\title{
Reciprocal heterospecific pollen interference among alien and native species
}

\author{
Eva M. Malecore ${ }^{1}$, Sylvie Berthelot ${ }^{2}$, Mark van Kleunen ${ }^{1,3} \&$ Mialy Razanajatovo $^{1}$ \\ ${ }^{1}$ Ecology, Department of Biology, University of Konstanz, Universitätsstrasse 10, D-78457 Konstanz, Germany \\ ${ }^{2}$ Albert-Ludwigs-Universität Freiburg, Department of Biometry and Environmental System Analysis, Freiburg im \\ Breisgau, Germany \\ ${ }^{3}$ Zhejiang Provincial Key Laboratory of Plant Evolutionary Ecology and Conservation, Taizhou University, Taizhou \\ 318000, China \\ *Correspondence: eva.malecore@uni-konstanz.de
}

\section{Abstract}

1. Heterospecific pollen interference has recently been proposed as a mechanism contributing to the success of alien invaders, as heterospecific pollen of alien plants interferes with the reproduction of natives by reducing fruit and seed set. However, no study has looked at the opposite interaction. Moreover, few studies have considered the roles of phylogenetic and trait distances between pollen donors and recipients.

2. We did a large multi-species experiment in which we used alien and native species both as pollen recipients and as pollen donors, and included phylogenetic as well as trait distance as explanatory variables.

3. We found that both alien and native recipients suffered from heterospecific pollen from donors of the opposite status in terms of seed and fruit set. Phylogenetic distance and trait distance both affected heterospecific pollen interference, but the effect depended on recipient and donor statuses.

4. We conclude that heterospecific pollen interference affects both native and alien recipients, thus indirectly altering community composition and increasing biotic resistance against invaders.

Keywords: Invasion ecology; Biotic resistance; Exotic plants; Heterospecific pollen; Reproductive interference; Alien plants; Indirect plant-plant interactions; Darwin's naturalization hypothesis. 


\section{Introduction}

Plants grow in communities, where they co-occur and interact continuously with other species. Co-flowering plant species can interact through pollinator sharing (e.g., Waser et al., 1996; Bascompte et al., 2003; Mitchell et al., 2009). Interspecific pollinator sharing can impact reproduction of the plants due to effects on visitation rates, and can result in heterospecific pollen (HP) transfer (Ashman and Arceo-Gómez, 2013). While HP transfer can result in a lower male fitness of the donor species due to loss of pollen (pollen discounting, Morales and Traveset, 2008), it can also result in a lower female fitness of the recipient species by reducing fruit and seed set. This so-called HP interference may affect recruitment and thus the persistence of a species in a community.

Heterospecific pollen interference occurs through a direct negative interaction on the pistil of the recipient species, where it can act at three levels: the stigma, the style and the ovule. At the stigma level, HP can mechanically clog the access to conspecific pollen (CP) or interfere with its adhesion and prevent its germination (Galen and Gregory, 1989). At the style level, HP tube growth can also cause clogging, or there can be allelopathic inhibition of CP tube growth (Arceo-Gómez and Ashman, 2013). At the ovule level, ovule usurpation, hybridization or inhibition of seed development are possible (e.g. Kanchan and Chandra, 1980; Thomson et al., 1981; Harder et al., 1993; Arceo-Gómez and Ashman, 2011). Thus, HP interference can affect fruit and seed set due to physical mechanisms as well as ineffective interspecies recognition. The importance of HP interference is likely to depend on the degree of (dis)similarity in floral traits between the recipient and donor. Moreover, if the plant species have a long history of co-occurrence, they may have evolved mechanisms to reduce HP interference, since HP interference would select for individuals less affected by it.

Recently, HP interference has gained interest for its role in plant invasions (Arceo-Gómez and Ashman 2016). In their new range, many alien invaders can use resident pollinators to reproduce (Traveset \& Richardson 2014; Razanajatovo et al. 2015; Razanajatovo \& van Kleunen 2016). This implies that the alien forms novel interactions, including HP interference, with native and other alien plant species with whom it does not share a co-evolutionary history (Saul, Jeschke and Heger 2013). Indeed, previous studies have shown high magnitudes of pollinator sharing between alien and native plant species (Moragues \& Traveset 2005; Jakobsson et al. 2008), and alien pollen has been found on stigmas of native flowers in invaded communities (e.g. Jakobsson et al. 2008). To explain the success of alien species, many studies have focused on how HP from alien species affects native species (Arceo-Gómez and Ashman, 2013). In a meta-analysis, Arceo-Gómez and Ashman (2016) found that invasive HP donors reduced fruit- and seed-set of recipients to a greater degree than native HP donors did, suggesting that the negative impacts of invasive plants on natives had previously been 
underestimated (Arceo-Gómez and Ashman 2016). However, all recipients in previous studies were natives, thus it remains unclear whether there is any effect of native HP donors on alien recipients. Given that alien species have to integrate themselves into native plant-pollinator networks, it is very likely that they will receive a lot of HP from native plants. This is because the alien species will, at least initially, be in the minority and are likely to use generalist pollinators that also visit many native species. If native HP reduces the seed production of the alien species, this would indicate that HP interference could increase the biotic resistance of native communities against alien invaders.

Besides on the alien-native status of the recipient and donor, and thus their co-evolutionary history, the effect of HP might also depend on the phylogenetic relatedness of the donor and recipient species. Phylogenetic distance might play a role in determining HP interference since closely related species are more likely to have similar floral morphologies and interspecies recognition systems involved in mechanisms like stigma adhesion, style clogging, ovule usurpation and hybridization. Such an interspecies recognition system usually involves barrier and promotion genes active in the pistil, and penetration and reaction genes active in the pollen (Hogenboom, 1973). Such genes may be phylogenetically conserved, making it more likely that HP is incorrectly recognized as CP when two species are closely related. In line with this hypothesis, pre- and post-zygotic reproductive barriers have been found to increase with phylogenetic distance (Moyle et al., 2004; Scopece et al., 2008; Moyle and Nakazato, 2010). On the other hand, interference between less related species could increase due to increased allelopathy (Wilcock and Neiland, 2002; Carvallo and Medel, 2016). Therefore, it remains to be tested whether plant species that are phylogenetically closely related suffer more or less from HP interference (but see Arceo-Gómez and Ashman, 2016 and Arceo-Gómez et al., 2019).

Some morphological characteristics that could play a role in HP interference are style length and pollen size. Donor pollen could physically clog the stigma of the recipient, especially when donor pollen grains are smaller than recipient pollen grains, by impeding CP hydration and germination (Galen and Gregory, 1989; McLernon et al., 1996). Although larger pollen grains could block a larger area, they often cannot adhere to the papillae of smaller grained species (McLernon et al., 1996). Therefore, a larger difference in pollen size would lead to a mismatch between pollen and stigmapapillae size (Ashman and Arceo-Gomez, 2013). Longer styles are more likely to act as a barrier to HP, as pollen tubes are less likely to reach the ovule (Smith-Huerta, 1997; Travers and Shea, 2001; Mazer et al., 2010). In addition, pollen coming from donor species with longer styles than the recipient, might clog the style of the recipient species more easily, since its pollen produces longer 
tubes. Thus a recipient with a style shorter than the donor might be more susceptible to HP interference (Ashman and Arceo-Gomez, 2013).

We conducted experimental hand pollinations that included both alien and native recipients as well as alien and native HP donors. Due to a non-shared evolutionary history at the community level, we expected HP to affect species independently of recipient status, but with a higher susceptibility to opposite status donors. Furthermore the native species used in this study are naturally co-occurring species whereas the aliens are not, we expected native recipients to suffer less from native donors, and alien recipients to suffer strongly from both native and alien donors. Additionally, we determined phylogenetic and trait (style length, pollen size) distances to examine their effects on HP interference. We expected the effect of phylogeny to be either positive or negative. We presumed there would be a higher susceptibility when the traits were more dissimilar, thus we expected HP interference to increase with trait distance. Specifically, we asked the following questions: 1) Does HP overall negatively affect fruit and seed set? 2) Does HP interference depend on similarity in the alien-native status (opposite or same) of the donor and recipient plants? 3) Does HP interference depend on phylogenetic and trait distances between the donor and recipient plants?

\section{Material and Methods}

\section{Study species}

To test for potential HP interference effects on both alien and native species, we selected 20 species, of which nine are alien and 11 are native to Germany (Error! Reference source not found. in Supporting Information), and which cover a broad range of phylogenetic and floral trait distances. To distribute the work load, we divided the species into two groups, one of 12 species and one of 8 species, that were grown in winter 2016 and summer 2017, respectively. All 20 species are insectpollinated forbs, and were selected as they occur in similar habitats (mostly grasslands and ruderal sites) and have overlapping flowering times (Kühn, Durka \& Klotz 2004, Error! Reference source not found.). Thus heterospecific pollination between those species was plausible in natural conditions. Most species were grown from seeds, but four were collected as seedlings in the area of Konstanz, Germany (Error! Reference source not found.). The seeds were sown in trays, and the resulting seedlings were transplanted into bigger pots. Pot sizes ranged from 1 to $3 \mathrm{~L}$ depending on species size, and were positioned in pollinator-free greenhouse compartments (see Error! Reference source not found. for details on greenhouse conditions) of the Botanical Garden of the University of Konstanz, Germany. For each species, we had approximately 60 plants to ensure that we would have enough flowering plant material for each treatment (Error! Reference source not found.). Since the 
species varied in their rate of development, the fast growing ones were trimmed to delay their flowering, so that more species would flower simultaneously.

\section{Experimental set-up}

The first group of species flowered from November 2016 to March 2017, and the second group of species from April 2017 to August 2017. To test for HP interference, we manipulated HP and CP deposition using hand pollination, and assessed fruit and seed set of the pollinated flowers. First, we assessed for each species the average number of pollen grains per anther. Therefore, we collected a fixed number of anthers and extracted the ripe pollen grains by shaking them in a Coulter-Isoton-IIdiluent-buffer solution, and counting the number of pollen grains with a Coulter Counter Multisizer 3 (Beckman Coulter, Krefeld, Germany). Then, based on the average number of pollen grains per anther of each species, we prepared pollen mixtures by combining an approximately equal amount of heterospecific and conspecific pollen for each HP treatment. We chose a 1:1 ratio to increase the comparability to other studies on HP interference (see Arceo-Gomez \& Ashman 2016). For each pollen mixture, we used anthers from one conspecific individual and one heterospecific individual. To avoid self-pollination, the conspecific donors were always a different individual than the pollen recipient (in some rare cases, we used multiple individuals as donors due to insufficient numbers of flowers on each donor). We released the pollen from the anthers by holding each anther with a tweezer, and tapping the anther on a clean surface. Then, we mixed the con- and heterospecific pollen on the clean surface using tweezers or toothpicks. We used toothpicks or tweezers to apply this mixture in saturating amounts on the stigmas of three to five flowers of the recipient plant, and we did this for three to five individuals per species combination. As a control, we applied conspecific pollen only with a comparable amount to the HP treatment on three to five flowers of three to five individuals per species. For each treatment, we applied an amount large enough to have pollen saturation. Each individual received only one of the two pollen-treatment types. To avoid selfing, we removed the anthers of recipient flowers before dehiscence. Prior emasculation could not be done on Nicandra physalodes and Lotus corniculatus, since buds would not survive emasculation. Therefore we removed the anthers of the recipient flowers of these species immediately before pollination and before they could touch the stigmas. Within the two groups of species, we aimed to use all species both as donors and recipients. However, as some species did not flower simultaneously in our experiment, we obtained 145 out of 208 possible combinations (Error! Reference source not found.). After ripening, the seeds produced by each treated flower were collected and counted using a seed counter (Pfeuffer Contador Seed Counter, Germany) or by hand. 


\section{Phylogenetic and floral-trait distance measures}

To test whether the degree of HP interference is associated with the phylogenetic distance and the floral trait distance between the species, we constructed a phylogenetic tree for our species and measured pollen size and style length for each species (Error! Reference source not found.). The phylogenetic tree was constructed by pruning a modified version (Malecore et al. 2018) of the dated DaPhnE supertree of Central European plant species (Durka \& Michalski, 2012). For each recipientdonor pair, we calculated the phylogenetic distance using the cophenetic function of the "ape" package (Paradis et al. 2004) in R (R-Core-Team, 2016).

We expected that HP interference depended on the size of the pollen and the length of the style, thus we measured the average pollen size $(\mu \mathrm{m})$ and style length $(\mathrm{mm})$ for each species. For pollen size, we randomly selected ten anthers each from three individuals per species, and took the midpoint of the range of sizes measured in the Coulter Counter. We used a digital caliper to measure the length of the style on one flower from ten individuals per species. For each recipient-donor pair, we used the function dist in $\mathrm{R}$ to calculate the joint (multivariate) Euclidean trait distance based on pollen size and style length.

\section{Statistical analysis}

We firstly tested whether there was HP interference and whether it depended on the status of the recipient and donor species. Secondly, we tested whether the degree of HP interference depended on the phylogenetic and floral-trait distances between the recipient and donor species. As response variables, we used fruit set, where a flower was counted as having set fruit when it produced at least one seed, and seed set (i.e. the number of seeds per fruit). Note, the analyses of seed set did not include flowers that did not produce any seeds. This was done to avoid zeroes when calculating the effect size as log response ratio, since adding a constant can result in a large over-estimation of the effect size (Rosenberg, Rothstein, \& Gurevitch, 2013). Fruit and seed set were averaged per plant individual.

\section{Effect of species status on HP interference}

To test whether HP affected fruit set and seed set, and whether this depended on the status of the pollen recipient and donor species, we used linear hypothesis testing and multiple comparisons for parametric models with a custom set contrast matrix, using the glht function of the "multcomp" package ("single-step" adjustment, Hothorn et al, 2008) (Table 1). For the analysis of fruit set, we used a binomial Generalized Linear Mixed Model (GLMM) implemented using the glmer function of the "Ime4" package (Bates et al. 2015). For the analysis of seed set (for the subset of flowers that had produced fruits), we log transformed the response variable to use a parametric model, and used a Linear Mixed Model (LMM) using the lmer function in the "lme4" package. In those models, we 
used as explanatory variable a dummy factor with six levels: (1) CP-only treatment for native recipient species, (2) HP treatment for native recipient and native donor species, (3) HP treatment for native recipient and alien donor species, (4) CP-only treatment for alien recipient species, (5) HP treatment for alien recipient and alien donor species, and (6) HP treatment for alien recipient and native donor species. To account for non-independence among data points from the same donor species, from the same recipient species, and from the same dataset (i.e. the two groups of species grown at different times), we included donor species, recipient species and dataset as random factors. Using a custom set contrast matrix for the dummy factor (Table 1), we tested first whether fruit and seed set (a) differed between native and alien recipient species, and (b) was overall lower after heterospecific than after conspecific-only pollination (i.e. whether there is HP interference). Second, we tested whether the magnitude of HP interference depended on (c) recipient status and (d) whether the recipient and donor had the same or opposite species status. Finally, we tested whether recipient status determined (e) the effect of having opposite species status and (f) having the same species status on HP interference.

\section{Effect of phylogenetic and floral-trait distances on HP interference}

We assessed the phylogenetic signal for pollen size and stigma length by calculating Pagel's $\lambda$ with the phylosig function of the "phytools" package (Revell 2012). To test how phylogenetic and floraltrait distances between recipient and donor species affected fruit set after heterospecific pollination, we used a binomial GLMM implemented in the glmer function of the "lme4" package. As seed set varied largely among species, we first normalized it by calculating for each individual plant the logresponse ratio of the average number of seeds per fruit in the HP treatment and the average number of seeds per fruit in the CP treatment of the respective species. The log-response ratio of seed set was then analysed using a Gaussian LMM implemented in the lmer function of the "Ime4" package. We first ran a model including the phylogenetic distance only, both as linear and quadratic terms, to test for potential nonlinear effects. We did the same using trait distance. In a second set of models, we used either phylogenetic or trait distance as the explanatory variable. Here we also included recipientstatus (alien and native) and a dummy factor indicating whether or not the donor was of the same or opposite status as factors, as well as the two-way interactions of the distance measures with recipientstatus and the dummy factor, and the three-way interaction. To account for non-independence among data points from the same donor species, the same recipient species, and from the same dataset, we included donor species, recipient species and dataset as random factors. We used a Bayesian approach to plot the $95 \%$ credible intervals in the figures. 
We performed additional analyses using style length and pollen size separately. Here, the difference of the style length or of pollen size between recipient and donor species was used as the explanatory variable, and the same model structure as for the joint trait distance was used (Error! Reference source not found.-9 and Error! Reference source not found.-4).

\section{Results}

We treated 1702 flowers, of which 1305 set fruit (679 out of 872 for the first dataset, and 626 out of 830 for the second dataset). Seed set ranged from 1 to 4968, depending on the recipient species (with Papaver somniferum having the highest seed number), and an overall median of 13 seeds.

A
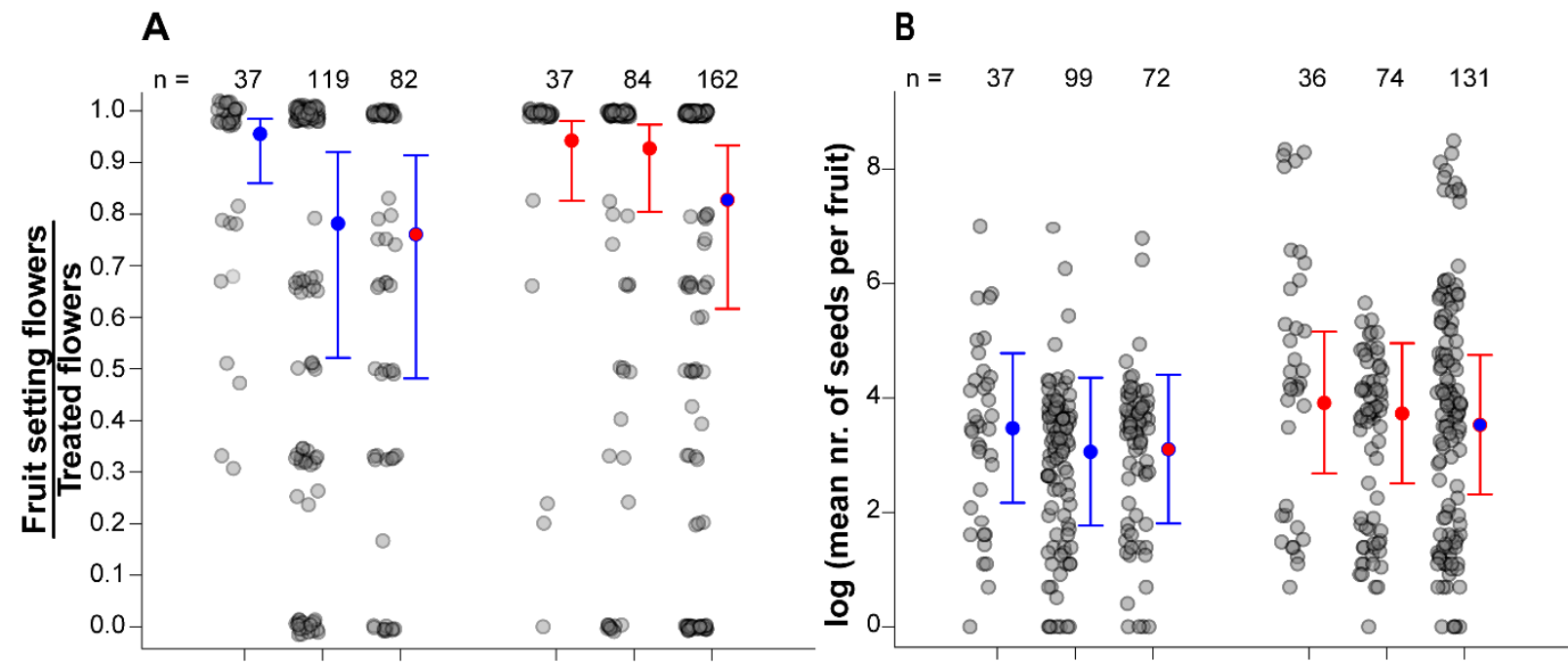

CP HP HP $\quad$ CP HP HP

Pollen type

: alien recipient, alien donor - alien recipient, native donor - native recipient, native donor

Pollen type

Figure 1 Plot showing the fitted values (with standard error) for the different pollination treatments, for fruit set expressed as the proportion of fruit setting flowers on treated flowers (A) and seed set expressed as the natural log of the number of seeds per fruit per plant (B). CP: conspecific pollen treatment. HP: heterospecific pollen treatment. Blue outlines: native recipient. Blue filling: native donor. Red outlines: alien recipient. Red filling: alien donor. The $n$ indicates the number of data points per category. Grey dots: data points per individual plant.

\section{HP reduced fruit and seed set and was affected by recipient and donor status}

Overall, HP significantly reduced both fruit set and seed set (Table 1, Figure 1). Compared to the CP treatment, the HP treatment reduced the likelihood of fruit set from $89 \%$ to $74 \%$, and the number of seeds per fruit by $53 \%$. HP of a species with the same status as the recipient reduced fruit set in the case of native recipients only, whereas HP of a species with the opposite status reduced fruit set independently of recipient status (Table 1, Figure 1). In other words, HP reduced fruit set for each combination of donor and recipient status, except when both species were alien. For seed set, however, HP interference did not depend on the status of the recipient and donor species (Table 1, Figure 1). 


\section{Phylogenetic and trait distances interacted with species status}

In the models that did not consider the status of the species, there were no significant linear and nonlinear effects of phylogenetic distance on HP interference in terms of fruit set and seed set (Error!

Reference source not found.). However, models that also included the status of the species had a better fit for fruit set $(\triangle \mathrm{AIC}>5)$, and the main effect of phylogenetic distance was not significant. Nonetheless, HP interference in terms of fruit set decreased (i.e. fruit set increased) significantly with increasing phylogenetic distance between donor and recipient for alien recipients, but not for natives, as indicated by the significant phylogenetic distance $\times$ recipient-status interaction (Table 2, Figure 2). In addition, the model showed that HP interference was overall stronger when the HP came from opposite status donors. HP interference in terms of seed set was overall slightly lower for native recipients than for alien recipients, but phylogenetic distance had no significant effects (Table 2 , Figure

2).

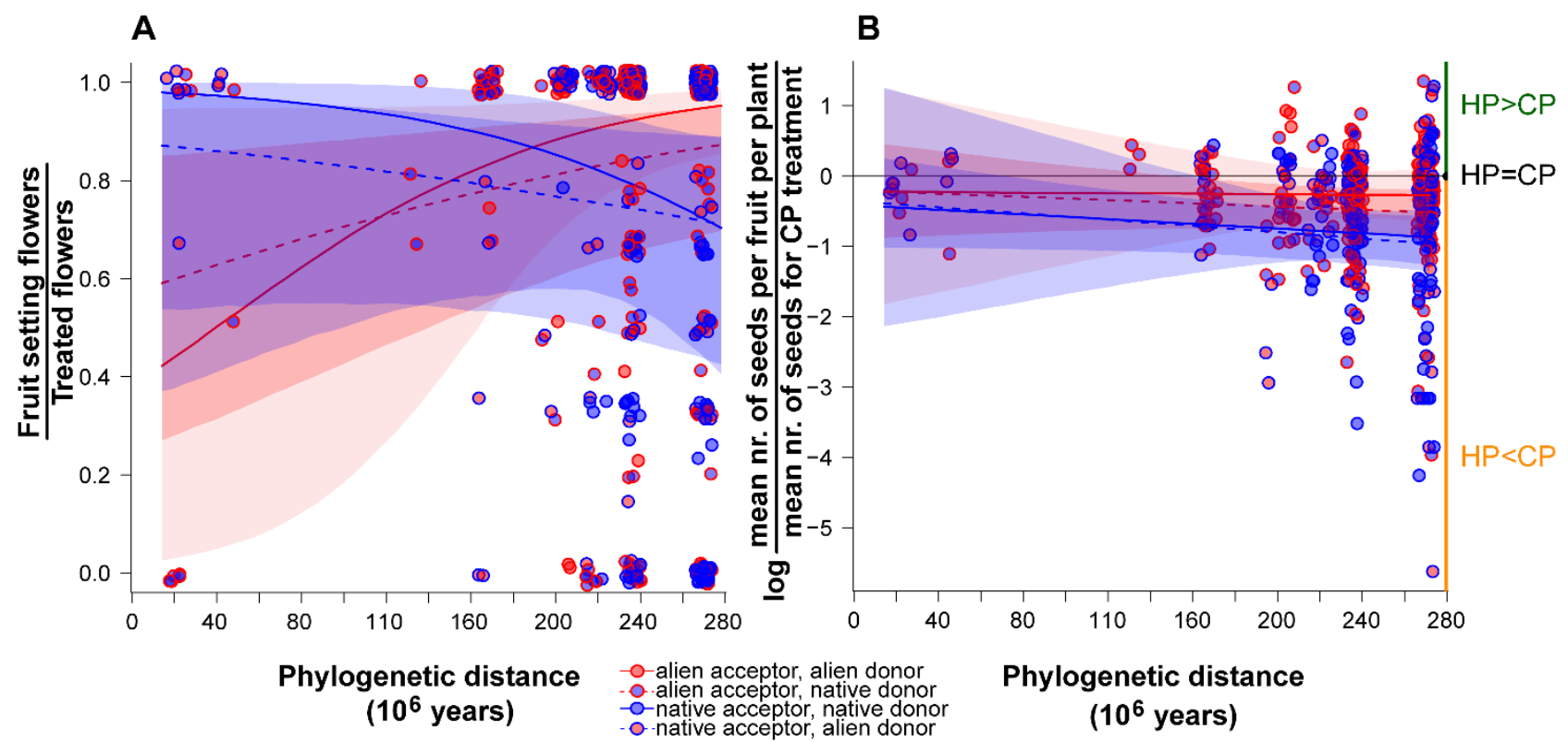

Figure 2 Data points for each individual plant receiving HP treatment and fitted lines (with 95\% credible intervals) for the binomial and Gaussian models including phylogenetic distance between pollen donor and recipient species (million years) as the explanatory variable, for fruit set (A) and seed set (B). Seed set is expressed as the natural log of the ratio of the mean number of seeds per plant and the mean number of seeds for conspecific pollen treatment. Blue solid line and blue dots: native recipient with native donor. Blue dotted line and blue dots with red background: native recipient with alien donor. Red solid line and red dots: alien recipient with alien donor. Red dotted line and red dots with blue background: alien recipient with native donor. CP: conspecific pollen treatment. HP: heterospecific pollen treatment.

Among our 20 study species, there was no significant phylogenetic signal for pollen size (Pagel's $\lambda=6.12 \times 10^{-5}$ ) and style length (Pagel's $\lambda=6.61 \times 10^{-5}$ ). In the models that did not consider the status of the species, there were no significant linear and non-linear effects of trait distance on HP 
interference in terms of fruit set and seed set (Error! Reference source not found.). However, models that also included the status of the species had a better fit (lower AIC values), and the main effect of trait distance was not significant. Nonetheless, HP interference in terms of fruit set increased with HP from opposite status donors, and decreased significantly with increasing trait distance for recipients treated with opposite status donors only, as indicated by the significant trait distance $x$ opposite-donor-status interaction (Table 3, Figure 3). HP interference in terms of seed set decreased with increasing trait distance for native recipients receiving HP from opposite status donors, and generally tended to be higher for native recipients than for alien recipients, but this effect was only marginally

significant.

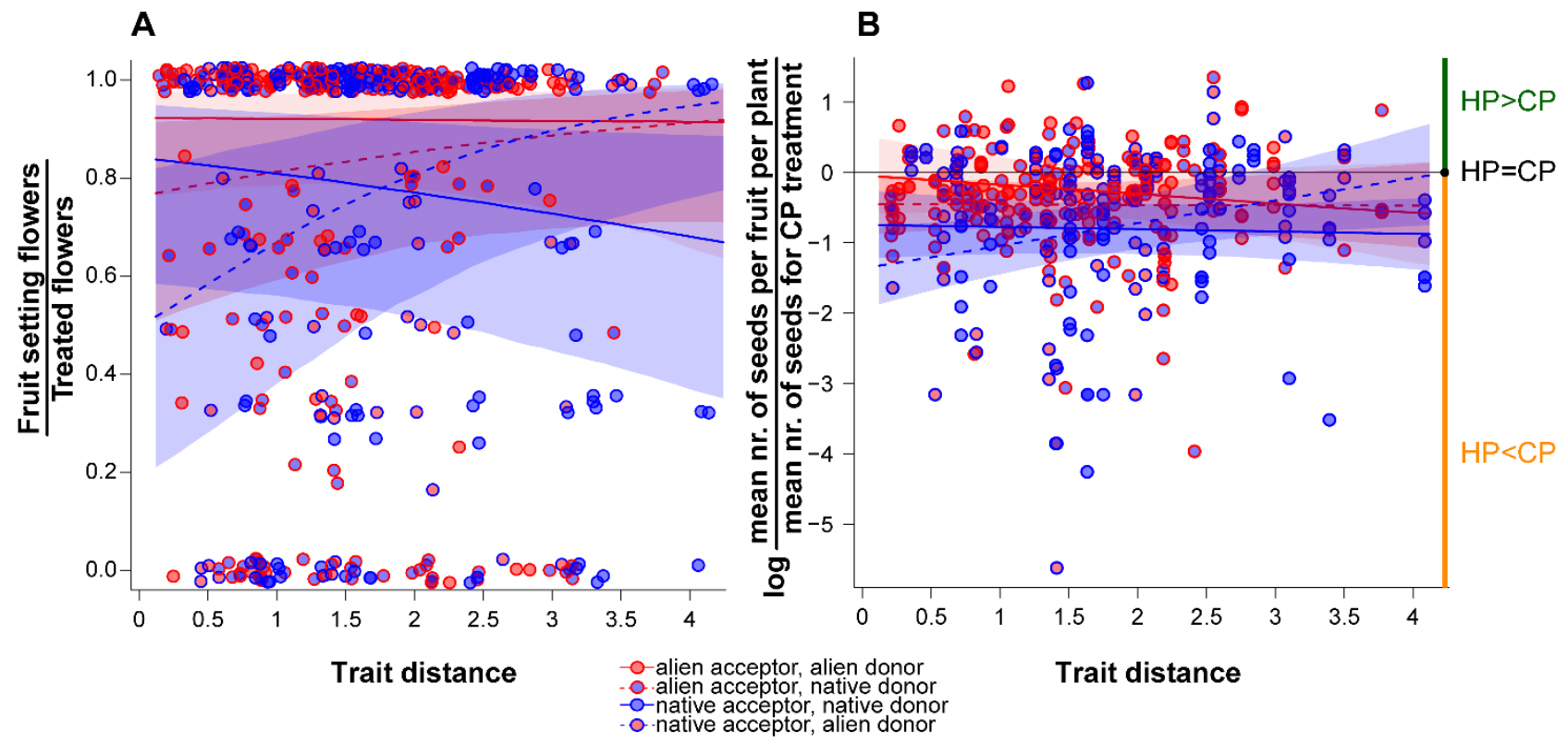

Figure 3 Data points for each individual plant receiving HP treatment and fitted lines (with $95 \%$ credible intervals) for the binomial and Gaussian models including trait distance as the explanatory variable, for fruit set (A) and seed set (B). Seed set is expressed as the natural log of the ratio of the mean number of seeds per plant and the mean number of seeds for conspecific pollen treatment. Blue solid line and blue dots: native recipient with native donor. Blue dotted line and blue dots with red background: native recipient with alien donor. Red solid line and red dots: alien recipient with alien donor. Red dotted line and red dots with blue background: alien recipient with native donor. CP: conspecific pollen treatment. HP: heterospecific pollen treatment.

\section{Discussion}

Using a large multi-species experiment, we provide the first evidence that HP not only has negative effects on fruit and seed set of native species, but also of alien species. In particular, HP interference was stronger for aliens if the pollen came from a native instead of from another alien species. HP interference did not depend directly on phylogenetic or trait distance between donor and recipient, but showed complex interactions of the distance measures with recipient and donor status. Thus it 
remains a challenge to predict HP interference from phylogenetic and trait distances. However, our results indicate that HP interference can not only increase the impacts of aliens on natives, but also can increase biotic resistance of native communities against alien invaders.

Table 1 Linear hypothesis testing and multiple comparisons with a custom-set contrast matrix for fruit set and seed set. For fruit set, a binomial Generalized Linear Mixed Model (GLMM) was used. For seed set (for the subset of flowers that had produced fruits), a Linear Mixed Model (LMM) was used after log-transforming the response variable. As the explanatory variable a dummy factor with six levels was used: (1) conspecific-pollen-only treatment for native recipient species, (2) heterospecific-pollen treatment for native recipient and native donor species, (3) heterospecificpollen treatment for native recipient and alien donor species, (4) conspecific-pollen-only treatment for alien recipient species, (5) heterospecific-pollen treatment for alien recipient and alien donor species, and (6) heterospecific-pollen treatment for alien recipient and native donor species. Donor species, recipient species and dataset were included as random factors. For custom contrast matrix used see Error! Reference source not found..

\begin{tabular}{|c|c|c|c|c|}
\hline \multirow{2}{*}{ Linear hypotheses } & \multicolumn{2}{|c|}{ Fruit Set } & \multicolumn{2}{|c|}{ Seed Set } \\
\hline & Estimate (SE) & $\mathbf{P}(>|\mathrm{z}|)$ & Estimate (SE) & $\mathbf{P}(>|\mathbf{z}|)$ \\
\hline $\begin{array}{l}\text { Fruit or seed set higher for native than for alien } \\
\text { recipients }\end{array}$ & $-0.4796(0.783)$ & 0.9755 & $-0.5135(0.882)$ & 0.9805 \\
\hline $\begin{array}{l}\text { Fruit or seed set is higher for CP than for HP } \\
\text { treatment }\end{array}$ & $1.3667(0.259)$ & $<0.001$ & $0.3380(0.109)$ & 0.0104 \\
\hline HPI higher for native than for alien recipients & $0.5857(0.289)$ & 0.1903 & $0.0530(0.116)$ & 0.9933 \\
\hline $\begin{array}{l}\text { Fruit or seed set in HP treatment is higher with } \\
\text { same status than with opposite status donors }\end{array}$ & $0.5719(0.202)$ & 0.0228 & $0.0772(0.095)$ & 0.9223 \\
\hline $\begin{array}{l}\text { HPI in HP treatment with opposite status donors } \\
\text { higher for native than for alien recipients }\end{array}$ & $0.3608(0.374)$ & 0.8549 & $-0.0076(0.137)$ & 1.0000 \\
\hline $\begin{array}{l}\text { HPI in HP treatment with same status donors } \\
\text { higher for native than for alien recipients }\end{array}$ & $0.8106(0.272)$ & 0.0148 & $0.1136(0.121)$ & 0.8682 \\
\hline
\end{tabular}

\section{The effect of alien and native status on the HP interference}

Although this effect was not significant in the main analyses (Table 1), native recipients appeared more susceptible than alien recipients, since fruit set was affected by both alien and native donors for native recipients, but by native donors only for alien recipients (Figure 1; Table 1). Moreover, in the analyses that included phylogenetic or trait distances, the log response ratio of the seed set of plants in the HP treatment to the average seed set of the species in the CP treatment was lower for native recipients than for alien ones (Figure 2-3; Table 2-3). In other words, alien recipients tended to suffer less from HP interference than native recipients did. In the short term, a reduction in fruit and seed set can lead to reduced recruitment and a shrinking of populations, and thus to a replacement of natives by aliens in invaded communities. In the long term, considering the variability 
in the sensitivity to HP among individuals of the same species (Error! Reference source not found.), one could expect a selection of genotypes less prone to HP, potentially resulting in an equilibrium of native species with the alien invaders, rather than a displacement.

Table 2 Binomial and Gaussian models including phylogenetic distance between pollen donor and recipient species as the explanatory variable, for HP interference measured based on fruit set and seed set. Recipient status (alien or native) as well as donor status (same-status or opposite-status) are included both as factors and as interactions. Seed set is expressed as the natural log of the ratio of the mean number of seeds per plant and the mean number of seeds for conspecific pollen treatment. P-values were calculated by comparing models with and without the factor in question.

\begin{tabular}{|c|c|c|c|c|}
\hline Phylogenetic distance (PD) & \multicolumn{2}{|c|}{ Fruit Set } & \multicolumn{2}{|c|}{ Seed Set } \\
\hline Parameter & Estimate & $\mathbf{P}$ & Estimate & $\mathbf{P}$ \\
\hline Fixed terms & $\left(\mathrm{SE}^{*}\right)$ & $\left(>X^{2}\right)$ & (SE) & $\left(>X^{2}\right)$ \\
\hline Intercept & $2.4202(0.565)$ & 0.0057 & $-0.2604(0.177)$ & 0.1478 \\
\hline $\mathrm{PD}$ & $0.6716(0.374)$ & 0.1355 & $-0.0060(0.168)$ & 0.09766 \\
\hline Recipient status (native) & $-1.0259(0.816)$ & 0.2709 & $-0.5372(0.253)$ & 0.04384 \\
\hline Donor status (opposite) & $-0.7715(0.338)$ & 0.0114 & $-0.2074(0.146)$ & 0.1179 \\
\hline Recipient status $\times$ Donor status & $0.4625(0.588)$ & 0.2262 & $0.1401(0.233)$ & 0.5278 \\
\hline PD $\times$ Recipient status & $-1.2690(0.609)$ & 0.0064 & $-0.0785(0.249)$ & 0.5715 \\
\hline PD $\times$ Donor status & $-0.3636(0.378)$ & 0.8600 & $-0.0538(0.177)$ & 0.7536 \\
\hline PD $\times$ Recipient status $\times$ Donor status & $0.7639(0.608)$ & 0.2117 & $0.0276(0.261)$ & 0.9167 \\
\hline Random terms & Standard de & lation & Standard de & iation \\
\hline Dataset & 0.000 & & 0.000 & \\
\hline Recipient Species & 1.428 & & 0.155 & \\
\hline Donor Species & 0.468 & & 0.022 & \\
\hline$A I C$ & 952.8 & & 951.8 & \\
\hline
\end{tabular}

Surprisingly, native recipients did not show an evolutionary adaptation to HP of co-occurring native donors, as they suffered equally from native and alien HP in terms of fruit set. Moreover, although we expected alien recipients to suffer equally from HP of native and alien donors, as they lack a coevolutionary history with both, they were most affected by HP from native species. The finding that aliens do not suffer from other aliens implies that it may be easier for new aliens to establish 
themselves if the community is dominated by other co-flowering aliens, in line with the invasional meltdown hypothesis (Simberloff \& von Holle, 1999). These findings suggest that in our study, native Central European species had strong negative effects when used as HP donors on both native and alien recipients. It is unclear why this is the case, and further research is required. However, it could be one of the many factors contributing to the overrepresentation of European species in the global naturalized alien flora (van Kleunen et al. 2015).

Although we cannot conclude that long coevolutionary histories of species reduce their HP interference, we demonstrated that the status of the donor matters, at least for the alien recipients. We found that the negative effect on natives did not depend on donor status, which contradicts previous studies (Arceo-Gomez and Ashman's meta-analysis, 2016). This could be because the meta-analysis included mainly invasive alien species, whereas in our study we focused on naturalized alien species that are not necessarily invasive. Most of the alien species used in our study are naturalized within the range of the natives, but it is unclear if all alien species are co-occurring with each other in their naturalized range. Assuming that aliens had a relevant coexisting time with the natives, i.e. a sufficient time to evolve various ecological and evolutionary responses to minimize HP interference, but not with the other aliens, co-occurrence seems to increase the negative effects of HP, contrary to expectation. Thus we can conclude that there is no evidence that a long coevolutionary history of species has reduced their HP interference.

Pollen deposition on the stigma of another species may result in pollen discounting for the donor, however our results indicate that such pollen is not entirely wasted because it may reduce the reproduction of competing plant species. This would particularly be the case if the donor species itself does not strongly suffer from HP interference. Having a detrimental effect as a HP donor could allow plants to compete not only with immediate neighbours, but also to affect other species elsewhere in the same community or, when the pollinators fly large distances, even in other communities. This function of pollen as a gametophytic weapon (Ashman and Gomez, 2013) has been rarely tackled, but could be a major selective force in the evolution of allelopathic pollen (i.e. pollen that releases chemicals that inhibit other pollen). Allelopathic chemicals inhibiting CP germination can even act in small doses, and thus require only a small amount of HP pollen (e.g., Sukhada and Jayachandra, 1980; Thomson et al., 1981, Murphy 2000), making it an effective weapon. The importance of allelopathy relative to other mechanisms such as style and stigma clogging, however, remains unknown. 


\section{The effect of phylogenetic distance and trait distance on HP interference}

While HP interference overall did not appear to be affected in a linear or non-linear way by phylogenetic and trait distances between the donor and recipient species, this was not the case when we also considered the status of the species. For alien recipient species, HP interference on fruit set decreased with phylogenetic distance. This is in line with Darwin's naturalization hypothesis, which poses that alien species can more easily naturalize when there are no close relatives in the recipient community (Procheş et al. 2008). However, for native recipient species, this was not the case, and there was even a tendency for a slight increase in HP interference on fruit set with increased phylogenetic distance. HP interference in terms of seed set, on the other hand, tended to be negatively affected by phylogenetic distance, even if only marginally significantly. Thus, the relationship between phylogenetic distance and HP interference depends on the status of the species and on the fitness measure (fruit set vs. seed set).

Table 3 Binomial and Gaussian models including floral trait (pollen size and style length) distance between pollen donor and recipient species as the explanatory variable, for HP interference measured based on fruit set and seed set. Recipient status (alien or native) as well as donor status (same-status or opposite-status) are included both as factors and as interactions. Seed set is expressed as the natural $\log$ of the ratio of the mean number of seeds per plant and the mean number of seeds for conspecific pollen treatment. P-values were calculated by comparing models with and without the factor in question.

\begin{tabular}{|c|c|c|c|c|}
\hline Trait distance (TD) & \multicolumn{2}{|c|}{ Fruit Set } & \multicolumn{2}{|c|}{ Seed Set } \\
\hline Parameter & Estimate & $\mathbf{P}$ & Estimate & $\mathbf{P}$ \\
\hline Fixed terms & $\left(\mathbf{S E}^{*}\right)$ & $\left(>X^{2}\right)$ & (SE) & $\left(>X^{2}\right)$ \\
\hline Intercept & $2.4298(0.571)$ & 0.0062 & $-0.2705(0.178)$ & 0.1353 \\
\hline $\mathrm{TD}$ & $-0.0210(0.232)$ & 0.4999 & $-0.1150(0.118)$ & 0.6260 \\
\hline Recipient status (native) & $-1.1332(0.811)$ & 0.2839 & $-0.5303(0.253)$ & 0.0537 \\
\hline Donor status (opposite) & $-0.7575(0.320)$ & 0.0089 & $-0.1930(0.142)$ & 0.2626 \\
\hline Recipient status $\times$ Donor status & $0.6730(0.523)$ & 0.2961 & $0.1718(0.226)$ & 0.4607 \\
\hline TD $\times$ Recipient status & $-0.1766(0.273)$ & 0.7619 & $0.0864(0.140)$ & 0.0644 \\
\hline TD $\times$ Donor status & $0.2789(0.272)$ & 0.0048 & $0.1120(0.137)$ & 0.0247 \\
\hline TD $\times$ Recipient status $\times$ Donor status & $0.5492(0.406)$ & 0.1874 & $0.1934(0.192)$ & 0.3209 \\
\hline Random terms & Standard dev & ation & Standard der & ation \\
\hline Dataset & $2.782 * 10$ & & 0.000 & \\
\hline
\end{tabular}




\begin{tabular}{l|c|c} 
Recipient Species & 1.467 & 0.412 \\
Donor Species & $4.177 * 10^{-1}$ & 0.151 \\
\hline AIC & $\mathbf{9 5 3 . 2}$ & $\mathbf{9 4 7 . 5}$ \\
*SE: standard error. AIC: Akaike information criterion & &
\end{tabular}

For fruit set, HP interference was stronger when the trait distance, based on style length and pollen size, was larger, but only when the pollen donor and recipient had the same status. This was the pattern that we had expected. However, HP interference decreased with increasing trait distance when pollen donor and recipient had opposite statuses. The latter was also observed for seed set, where a negative effect of trait distance on HP interference was found for the combination of native recipients and alien donors. The traits used to calculate trait distance showed no phylogenetic signal, i.e. trait values were not more similar for more closely related species. Thus we can assume that style length and pollen size, are likely to follow different evolutionary patterns than the traits captured by phylogenetic distance, which we assume are being mainly connected to the species pollen-recognition system. Thus, we suggest that the patterns observed result from a different evolutionary response for the traits here analysed.

In this study, we did not look at the exact mechanisms responsible for the reduction of fruit set and seed set. The breeding system of recipient and donor species, i.e. their self-compatibility, may play a role in the outcome of HP interference. According to the "SI $\times$ SC" rule (Lewis and Crowe, 1958; Hiscock and Dickinson, 1993; Li and Chetelat, 2010; Bedinger et al., 2011), self-incompatible (SI) species reject pollen from self-compatible (SC) donors but not the other way around (Murphy 2000). When we tested for effects of breeding system, we found that the effect of HP interference was highest for donors of opposite breeding system, for both self-compatible and self-incompatible pollen donors (Error! Reference source not found.). Thus our study does not confirm the "SI $\times$ SC" rule. An alternative mechanism that could explain HP interference is the so-called mentor effect (de Nettancourt, 1977). In the mentor effect, a compatible or congruent pollen type acts as a "mentor" to allow incompatible or incongruent pollen to pass the species-recognition system of the stigma (Hogenboom, 1973), with possibility to lead to style clogging, allelopathic effects, ovule usurpation or hybridization, which could lead to HP interference. Nevertheless, the mechanisms underlying the patterns found here are not yet fully clear. 

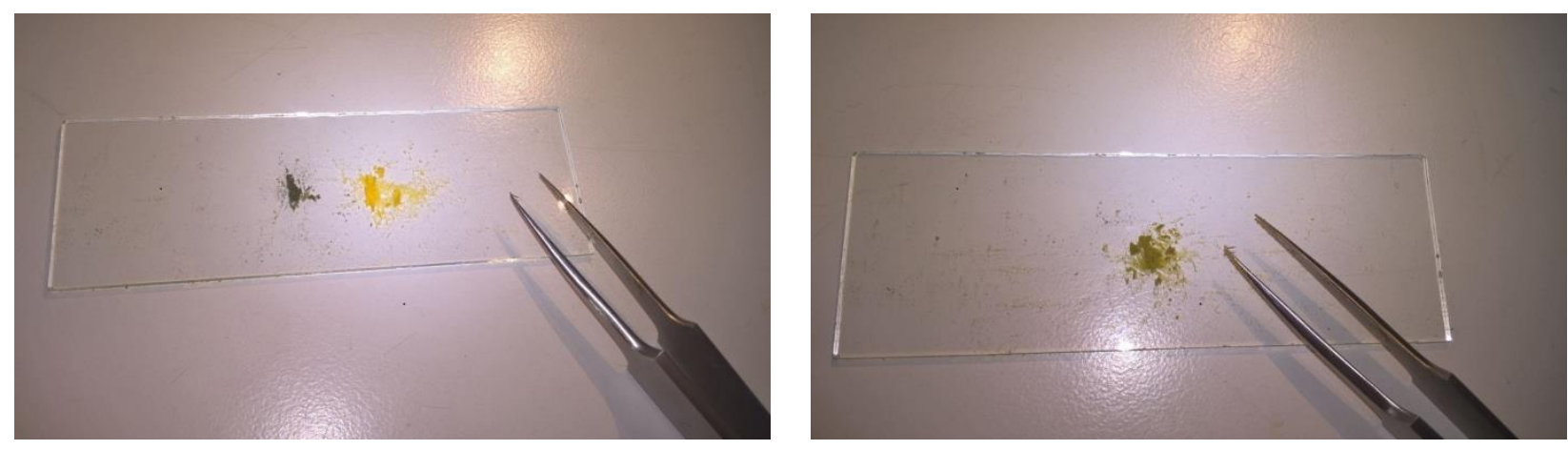

C

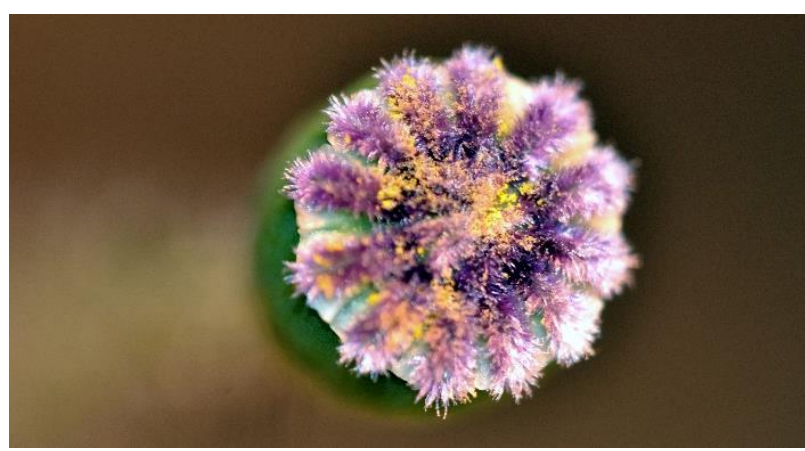

Figure 4 Preparation and application of heterospecific and conspecific pollen mix. The pollen of an heterospecific and of a conspecific donor are extracted from the anthers using tweezers (A) and then mixed on a glass slide (B). The pollen mix is then applied on the stigma of the donor species $(\mathrm{C})$

\section{Conclusion}

Overall, HP negatively affects fruit and seed set. However, HP interference depends on recipient and donor status, with native recipients suffering both from alien and native donors, and alien recipients suffering only from native donors. Unexpectedly, a long or non-existing coevolutionary history of species at community level could not explain this status-specific pattern. Rather, native and alien species seem to differ from each other for some unknown reason. Both phylogenetic distance and trait distance played a role in determining the effect size of HP interference, but this depended on the fitness measure used, affecting fruit set but not seed set, and on recipient and donor status. Our results suggest that traits related to post-pollination stages in the pollen-pistil interactions, such as style length and pollen size, also play a major role in HP interference.

This is the first study to show that HP interference matters for both native and alien recipients. Our multispecies study showed similarly strong negative impacts on the reproduction of natives whether the pollen donor was an alien or another native plant. Moreover, we showed that HP interference can increase the resistance of native communities against alien invaders. Thus we suggest that HP interference can indirectly affect community composition through its effects on reproduction. 


\section{References}

Arceo-Gómez, G. \& Ashman, T. L. (2011). Heterospecific pollen deposition: does diversity alter the consequences? New Phytologist, 192, 738-746.

Arceo-Gómez, G. \& Ashman, T. (2016). Invasion status and phylogenetic relatedness predict cost of heterospecific pollen receipt : implications for native biodiversity decline. Journal of Ecology, 104, 1003-1008.

Ashman, T.-L. \& Arceo-Gómez, G. (2013). Toward a predictive understanding of the fitness costs of heterospecific pollen receipt and its importance in co-flowering communities. Am. J. Bot., 100, 1061-1070.

Bascompte, J., Jordano, P. \& Melia, C. J. (2003). The nested assembly of plant - animal mutualistic networks, PNAS, 100(16), 9383-9387.

Bedinger, P. A., Chetelat, R. T., McClure, B., Moyle, L. C., Rose, J. K. C., Stack, S. M. et al. (2010) Interspecific reproductive barriers in the tomato clade : opportunities to decipher mechanisms of reproductive isolation, Sexual Plant Reproduction.

Bates, D., Maechler, M., Bolker, B. \& Walker, S., (2015). Fitting Linear Mixed-Effects Models Using lme4. Journal of Statistical Software, 67(1), 1-48.

Carvallo, G. O. \& Medel, R. (2016). Heterospecific pollen transfer from an exotic plant to native plants: assessing reproductive consequences in an Andean grassland. Plant Ecology \& Diversity, 9(2), 151-157.

Durka, W. \& Michalski, S. G. (2012). Daphne: a dated phylogeny of a large European flora for phylogenetically informed ecological analyses. Ecology, 93, 2297.

Galen, C. \& Gregory, T. (1989). Interspecific pollen transfer as a mechanism of competition: consequences of foreign pollen contamination for seed set in the alpine wildflower, Polemonium viscosum. Oecologia, 81, 120-123.

Harder, L. D., Cruzan, M. B. \& Thomson, J. D. (1993). Unilateral incompatibility and the effects of interspecific pollination for Erythronium americanum and Erythronium albidum (Liliaceae). Canadian Journal of Botany, 71(197 1), 353-358.

Hiscock, S. J. \& Dickinson, H. G. (1993). Unilateral incompatibility within the Brassicaceae : further evidence for the involvement of the self-incompatibility (S)-locus. Theoretical and Applied Genetics, 86, 744-753.

Hogenboom, N. G. (1973). Incongruity and incompatibility in intimate partner relationships. Wageningen: Centre for Agricultural Publishing and Documentation.

Hothorn, T., Bretz, F. \& Westfall, P. (2008). Simultaneous Inference in General Parametric Models. Biometrical Journal 50(3), 346--363. 
Jakobsson, A., Padrón, B., \& Traveset, A. (2008). Pollen transfer from invasive Carpobrotus spp. to natives-A study of pollinator behaviour and reproduction success. Biological Conservation, 141(1), 136-145.

Kanchan, S. \& Chandra, J. (1980). Pollen allelopathy - a new phenomenon. New Phytologist, 84, 739-746.

Kühn, I, Durka, W. \& Klotz, S. (2004). BiolFlor: a new plant-trait database as a tool for plant invasion ecology. Divers. Distrib., 10, 363-365.

Lewis, D., \& Crowe, L. K.(1958). Unilateral interspecific incompatibility in flowering plants. Heredity, 12: $233-256$

Li, W. \& Chetelat, R. T. (2010). A Pollen Factor Linking Inter- and Intraspecific Pollen Rejection in Tomato. Science, 330, 1827-1830.

Malecore, E. M., Dawson, W., Kempel, A., Müller, G. \& van Kleunen, M. (2018). Nonlinear effects of phylogenetic distance on early - stage establishment of experimentally introduced plants in grassland communities. Journal of Ecology, 1-13.

Mazer, S. J., Hove, A. A., Miller, B. S. \& Barbet-Massin, M. (2010). The joint evolution of mating system and pollen performance: Predictions regarding male gametophytic evolution in selfers vs outcrossers. Perspectives in Plant Ecol, Evol and Systematics 12(1), 31-41.

McLernon, S. M. \& Aarssen, W. (1996) Heterospecific pollen transfer between sympatric species in a midsuccessional old-field community. American Journal of Botany, 83(9), 1168-1174.

Mitchell, R. J. Flanagan, R. J., Brown, B. J., Waser, N. M. \& Karron, J. D. (2009) New frontiers in competition for pollination. Annals of Botany, 103, 1403-1413.

Moyle, L. C. \& Nakazato, T. (2010). Hybrid incompatibility "Snowballs" between Solanum species, Science, 329, 1521-1524.

Moyle, L. C., Olson, M. S. \& Tiffin, P. (2004) Patterns of reproductive isolation in three angiosperm genera. Evolution, 58(6), 1195-1208.

Moragues, E., \& Traveset, A. (2005). Effect of Carpobrotus spp. on the pollination success of native plant species of the Balearic Islands. Biological Conservation, 122(4), 611-619.

Morales, C. L. \& Traveset, A. (2008). Interspecific Pollen Transfer: Magnitude, Prevalence and Consequences for Plant Fitness. Critical Reviews in Plant Sciences, 27, 221-238.

Murphy, S. D. (2000). Field testing for pollen allelopathy. Journal of Chemical Ecology, 26(9), 21552172.

de Nettancourt, D. (1977). Incompatibility in Angiosperms. Springer Verlag, Berlin, Germany.

Paradis, E. Claude, J. \& Strimmer, K. (2004). APE: analyses of phylogenetics and evolution in R language, Bioinformatics, 20, 289-290. 
Procheş, S., Wilson, J. R. U., Richardson D. M. \& Rejmanek, M. (2008). Searching for phylogenetic pattern in biological invasions. Global Ecol. Biogeogr., 17, 5-10.

Razanajatovo, M., Föhr, C., Fischer, M., Prati, D., \& van Kleunen, M. (2015). Non-naturalized alien plants receive fewer flower visits than naturalized and native plants in a Swiss botanical garden. Biological conservation, 182, 109-116. .

Razanajatovo, M., \& van Kleunen, M. (2016). Non-invasive naturalized alien plants were not more pollen-limited than invasive aliens and natives in a common garden. Functional Ecology, 30(9), 1511-1520.

R-Core-Team (2016). R: A language and environment for statistical computing. $R$ Foundation for Statistical Computing. Vienna, Austria.

Revell, L. J. (2012). Phytools: An R package for phylogenetic comparative biology (and other things). Methods Ecol. Evol. 3 217-223.

Saul, W.C., Jeschke, J.M., \& Heger, T. (2013). The role of eco-evolutionary experience in invasion success. NeoBiota 17: 57-74.

Scopece, G., Widmer, A. \& Cozzolino, S. (2008) Evolution of Postzygotic Reproductive Isolation in a Guild of Deceptive Orchids, The American Naturalist, 171(3).

Simberloff, D. \& von Holle, B. (1999) Positive interactions of nonindigenous species: invasional meltdown?, Biological Invasions, (1), 21-32.

Smith-Huerta , N. L. 1997 . Pollen tube attrition in Clarkia tembloriensis (Onagraceae). International Journal of Plant Sciences 158, 519 - 52.

Thomson, J. D., Andrews, B. J., \& Plowright, R. C. (1981). The effect of a foreign pollen on ovule development in Diervilla lonicera (Caprifoliaceae). New Phytologist, 90(4), 777-783.

Travers, S. E. \& Shea, K. (2001). Individual variation, gametophytic competition and style length : does size affect paternity?, Evolutionary Ecology Research, 3, 729-745.

Traveset, A., \& Richardson, D. M. (2014). Mutualistic interactions and biological invasions. Annual Review of Ecology, Evolution, and Systematics, 45, 89-113.

van Kleunen, M. Dawson, W., Essl, F., Pergl, J., Winter, M., Weber, E. et al. (2015). Global exchange and accumulation of non-native plants. Nature, 525 .

Waser, N. M. Chittka, L., Price, M. V., Williams, N. M. \& Ollerton, J. (1996). Generalization in pollination systems and why it matters. Ecology, 77, 279-296.

Wilcock, C. \& Neiland, R. (2002). Pollination failure in plants : why it happens and when it matters, TRENDS in Plant Science, 7(6), 270-277. 